\title{
Mast cell activators: a new class of highly effective vaccine adjuvants
}

\author{
James B McLachlan ${ }^{1,5}$, Christopher P Shelburne ${ }^{1,5}$, Justin P Hart ${ }^{1}$, Salvatore V Pizzo ${ }^{1}$, Rajen Goyal ${ }^{1}$, \\ Rhea Brooking-Dixon ${ }^{1}$, Herman F Staats ${ }^{1-3}$ \& Soman N Abraham ${ }^{1,2,4}$
}

\begin{abstract}
Mast cells (MCs) have recently received recognition as prominent effectors in the regulation of immune cell migration to draining lymph nodes and lymphocyte activation. However, their role in the development of humoral immune responses is not clear. Here, we demonstrate that subcutaneous or nasal administration of small-molecule MC activators with vaccine antigens evokes large increases in antigen-specific serum immunoglobulin G (IgG) responses. These responses were MC dependent and correlated with increased dendritic cell and lymphocyte recruitment to draining lymph nodes. Nasal instillation of these formulations also evoked antigen-specific secretory IgA and provided protection against anthrax lethal toxin challenge in vitro and against vaccinia virus infection in vivo. Collectively, these results define the MC as an integral sensory arm of the adaptive immune system. Moreover, they highlight MC activators as a new class of vaccine adjuvants, capable of inducing protective antigen-specific immune responses through needle-free routes of administration.
\end{abstract}

Our understanding of MC function has been rapidly expanding in recent years. Although they had been principally thought of as a causative agent in allergy and asthma, MCs are now known to be essential for the resolution of bacterial infections through neutrophil mobilization to the site of infection ${ }^{1,2}$. MCs are also required for the enhanced recruitment of lymphocytes ${ }^{3}$ and Langerhans cells ${ }^{4}$ to draining lymph nodes (DLNs), a process required for the induction of adaptive immune responses ${ }^{5}$. Indeed, antigen-bearing dendritic cell (DC) migration from peripheral tissues into the DLNs is essential for the priming of cognate $\mathrm{T}$ cells and their subsequent ability to provide help for the production of antigen-specific antibodies by plasma B cells $s^{6,7}$.

Thus, MCs seem to coordinately initiate both the innate immune response and immune cell migration believed to be essential in stimulating the adaptive immune response. These functions are believed to be due to the capacity of MCs to rapidly release prestored inflammatory mediators such as tumor necrosis factor (TNF) and histamine within minutes of activation ${ }^{8}$. In the present study, we sought to examine the role of the MC in the induction of antigenspecific humoral immune responses. We hypothesized that the administration of small-molecule MC activating compounds in conjunction with protein antigens would result in a potent and protective antigenspecific immune response.

\section{RESULTS}

MC activators mobilize immune effectors to DLNs

MC-activating compounds comprise a structurally diverse, cationic group of peptides and polymeric compounds known to trigger $\mathrm{G}_{\mathrm{i}}$ protein-dependent exocytic release of MC granule components both in vitro and in vivo ${ }^{9,10}$. To characterize the ability of these compounds to initiate an immune response, we first examined the ability of several well known MC activators, including compound 48/80 (c48/80) ${ }^{11}$, MC degranulating peptide $(\mathrm{MCD})^{9}$ and catestatin $\left(\mathrm{CgA}_{344-364}\right)^{12}$ to mobilize elevated numbers of DCs and lymphocytes to DLNs. Subcutaneous footpad injection of c48/80 induced increases in both DC numbers and total cellularity in footpad DLNs that reached peak intensity by $24 \mathrm{~h}$ compared to vehicle-injected controls (Fig. 1). DC numbers and total cellularity then decreased by $48 \mathrm{~h}$ (data not shown). In order to determine the dependence of this response on MCs, we undertook this study in wild-type (WT) mice; MC-deficient $\left(\mathrm{Kit}^{\mathrm{W}} / \mathrm{Kit}^{\mathrm{W}-v}\right)$ mice, hereinafter referred to as $W / W^{v}$; and $\mathrm{W} / \mathrm{W}^{v}$ mice with MC-reconstituted footpads. Although initial footpad tissueresident DC numbers were identical in all strains (data not shown), $W / W^{v}$ mice demonstrated significantly lower cellularity and DC numbers in DLNs $24 \mathrm{~h}$ after c48/80 challenge compared to MC-sufficient mice (Fig. 1a,d,g). Similar MC-dependent increases in DCs and total cellularity were also observed with both MCD (Fig. 1b,e,g) and catestatin (Fig. 1 $\mathbf{1}, \mathbf{f}, \mathbf{g}$ ), in the same time course as induced by c48/80. Reduced DC trafficking to DLNs in $W / W^{v}$ mice could be explained, in part, by the reduced expression of the lymph node homing chemokine receptor CCR7 on $W / W^{v}$ footpad DCs compared to WT controls, $8 \mathrm{~h}$ after $\mathrm{c} 48 / 80$ challenge (Supplementary Fig. 1a,b online). This suggested a requirement for MCs in the optimal expression of CCR7 on DCs. In support of this, we found that prolonged incubation of bone marrow-derived DCs with c48/80 did not induce the expression of this migratory marker

Departments of ${ }^{1}$ Pathology, ${ }^{2}$ Immunology, ${ }^{3}$ Medicine and ${ }^{4}$ Molecular Genetics \& Microbiology, Duke University Medical Center, Durham, North Carolina 27710 , USA.

${ }^{5}$ These authors contributed equally to this work. Correspondence should be addressed to S.N.A. (soman.abraham@duke.edu).

Received 13 February; accepted 26 March; published online 20 April 2008; doi:10.1038/nm1757 

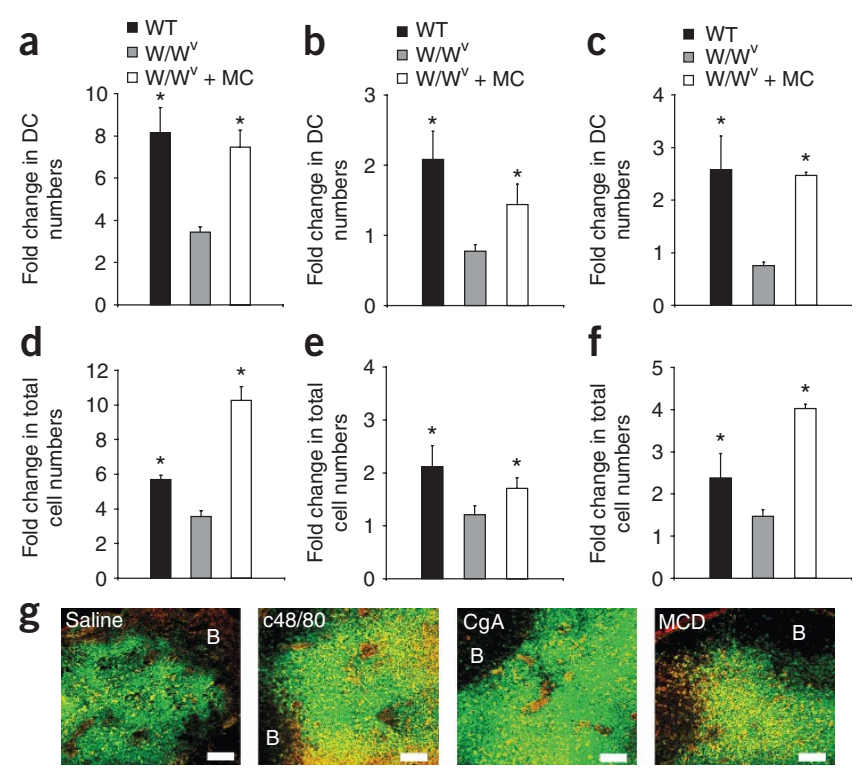

(Supplementary Fig. 1c). Collectively, these data suggest that MC-activating compounds such as c48/80 induce enhanced DC and lymphocyte trafficking to DLNs by activating MCs.

\section{c48/80 induces MC-dependent humoral immunity}

The ability of MC-activating compounds to coordinately regulate optimal increases in DC and lymphocyte sequestration in DLNs, events required for the initiation of adaptive immune responses ${ }^{5}$, suggested that protective antigen-specific immune responses could be generated through the administration of a MC activator together with a protein antigen. To test this notion, we immunized the footpads of WT mice with one injection of $0.5 \mu \mathrm{g}$ of PA (protective antigen from Bacillus anthracis) and $32 \mu \mathrm{g}$ of $\mathrm{c} 48 / 80$, the most potent of the MC activators tested. To demonstrate the direct contribution of MCs, we also immunized $W / W^{v}$ mice, as well as $W / W^{v}$ mice reconstituted with MCs in the footpad. Sera from each group of mice was collected on days 7 and 14 and assayed for PA-specific total IgG. PA alone elicited minimal responses in $\mathrm{WT}, W / W^{v}$ or MC-reconstituted $W / W^{v}$ mice by day 7 (data not shown). However, PA administered with c48/80 induced significant PA-specific IgG titers in WT mice by day 7, which increased by day 14 (Fig. 2a). PA administered with c48/80 in $W / W^{v}$ mice was able to induce an immune response, suggesting that $c 48 / 80$ can act on non-MCs. However, this response was significantly deficient at day 7 and day 14 compared to WT controls (Fig. 2a). Notably, this deficiency was repaired by the reconstitution of MCs at the site of injection (Fig. 2a). Finally, to confirm that $c 48 / 80$ was acting through DCs, as suggested by the data in Figure 1a-c, we made use of the CD11c-diphtheria toxin receptor (DTR) mouse that has a simian DTR under control of the murine CD11c promoter ${ }^{13}$. When these mice are injected with diphtheria toxin, their DCs are effectively ablated. We injected c48/80 plus PA into DC-ablated CD11c-DTR mice and unablated controls. As expected, DC-ablated CD11c-DTR mice were unable to mount an efficient PA-specific response compared to controls (Fig. 2b). The partial recovery of humoral responses on day 14 in CD11c-DTR mice is consistent with the fact that ablation of DCs only lasts $24-48 \mathrm{~h}$ (ref. 13). Therefore, these data collectively suggest that MC activators act as efficient adjuvants and that their mode of action is mediated through MCdependent enhancement of DC and lymphocyte migration to DLNs.
Figure $1 \mathrm{MC}$ activators induce DC mobilization and DLN hypertrophy. (a-f) Fold increase, over those in saline controls, in DC numbers (a-c) and total cell numbers (d-f) in DLNs of WT, W/WV and footpad MC-reconstituted $W / W^{v}$ mice $24 \mathrm{~h}$ after instillation of $32 \mu \mathrm{g}$ of $\mathrm{c} 48 / 80(\mathbf{a}, \mathbf{d}), 1 \mu \mathrm{M}$ catestatin (b,e) or $10 \mu \mathrm{M} \operatorname{MCD}(\mathbf{c}, \mathbf{f})$ into footpads. $n=4-6$ mice; * $P<0.05$ compared to $W / W^{V}$ mice. (g) Confocal images of representative DLN sections acquired from WT mice $24 \mathrm{~h}$ after saline, c48/80, MCD or catestatin $\left(\mathrm{CgA}_{344-364}\right)$ challenge in the footpads. Sections were stained with anti-CD3 for T cells (green) and anti-CD11c for DCs (red). Colocalization of DCs and T cells appears yellow. Similar results were obtained in three separate experiments. B, B-cell zone. Scale bar, $100 \mu \mathrm{M}$. Error bars, mean + s.d.

A major pre-stored component of the MC granule demonstrated to influence both lymphocyte and DC mobilization to DLNs is $\mathrm{TNF}^{3,14}$. Therefore, MC-derived TNF might be a critical component of the primary humoral response elicited by MC-activating compounds. To examine this, we repeated the previous study in the footpads of $W / W^{v}$ mice reconstituted with WT or $T n f^{-1-}$ MCs with $0.5 \mu \mathrm{g}$ of $\mathrm{PA}$ and $32 \mu \mathrm{g}$ of $c 48 / 80$. Once again, sera from each group of mice was collected after 7 and $14 \mathrm{~d}$ and assayed for PA specific total IgG. As expected, PA-specific responses were reduced in $W / W^{v}$ mice reconstituted with $\operatorname{Tnf}^{-1-}$ MCs compared to the responses observed in $W / W^{v}$ mice reconstituted with WT MCs after $7 \mathrm{~d}$ (Fig. 2c), indicating that MC TNF is a principal product in the development of optimal antigen-specific immune responses during MC activator challenge.

\section{c48/80 activates MCs in the NALT}

Although the previous results demonstrate that MC activation induces a humoral response after a subcutaneous injection, the primary route of pathogen entry is along mucosal surfaces, such as the gastrointestinal, urinary and respiratory tracts ${ }^{15}$. In light of this, we chose to induce an immune response by way of the nasal mucosa of mice through the activation of MCs with c48/80. Immunization at this site has been shown to effectively evoke antigen-specific serum-IgG as well as secretory-IgA (refs. 16-18) responses, presumably due to activation of the nasal associated lymphoid tissue (NALT) ${ }^{19}$. Initially, we characterized the MC populations in this region. Cross-sections through the nose revealed large numbers of MCs in close proximity to the NALT (Fig. 3a). These MCs showed a connective tissue (CTMC)
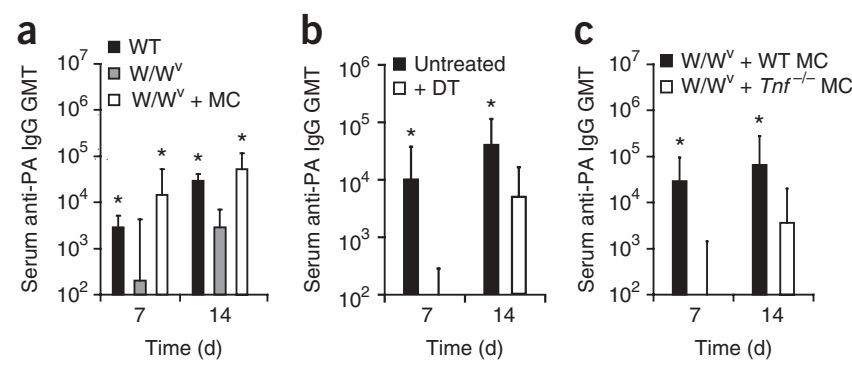

Figure 2 c48/80 induces MC-dependent humoral responses. (a) GMT of PA-specific serum IgG in WT, WIWV and footpad MC-reconstituted W/WV mice immunized with PA or with PA plus $c 48 / 80$. $n=6$ mice; ${ }^{*} P<0.05$ compared to WIWV mice. (b) GMT of PA-specific serum IgG in CD11c-DTR mice injected with saline (DC-sufficient mice) or diphtheria toxin (DCablated mice), immunized with PA or with PA plus $c 48 / 80$. $n=5-7$ mice; ${ }^{*} P<0.05$. (c) GMT of PA-specific serum IgG in W/Wv mice reconstituted with WT or $\mathrm{Tnf}^{-1-}$ MCs in the footpad, immunized with PA or with PA plus c48/80. $n=6$ mice. ${ }^{*} P<0.05$. Times on the $x$ axis are those of serum collection after immunization. Error bars, mean + s.d. 
a

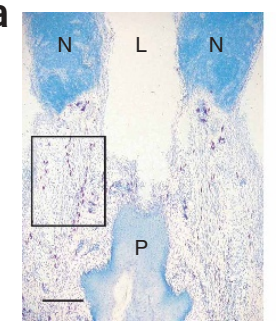

C

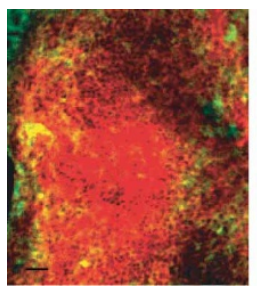

PA alone
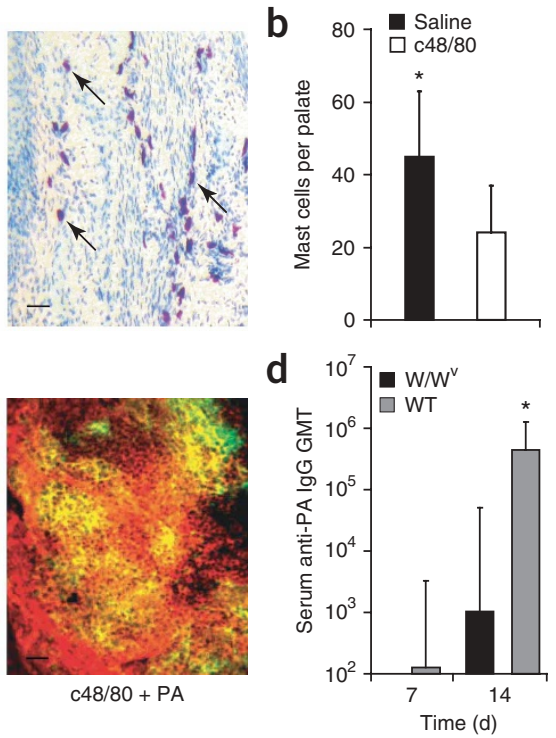

phenotype (as opposed to a mucosal phenotype (MMC) ${ }^{20}$ in their alcian blue/saffranin $\mathrm{O}$ staining properties (data not shown). This is noteworthy because CTMC are markedly more responsive to $c 48 / 80$ than $\mathrm{MMC}^{21,22}$. Delivery of $32 \mu \mathrm{g}$ of $\mathrm{c} 48 / 80$ into the nose induced a marked decrease in the numbers of MCs in or around the palate (Fig. 3b), probably owing to MC degranulation ${ }^{3}$. As was observed in the skin, administration of $\mathrm{c} 48 / 80$ induced DCs to migrate into the T-cell areas of the nasal associated lymphoid tissue (NALT) (Fig. 3c). In addition, instillation of c $48 / 80$ plus antigen into the nose was also found to induce antigen-specific $\mathrm{CD} 4^{+} \mathrm{T}$-cell activation as determined by antigen restimulation assays (Supplementary Fig. 2a online), but only modestly affected $\mathrm{CD}^{+}$T-cell responses (Supplementary Fig. 2b,c). Lastly, $14 \mathrm{~d}$ after nasal introduction of c $48 / 80$ in conjunction with PA, PA-specific serum IgG was increased in WT mice but not $W / W^{v}$ mice (Fig. 3d). No serologic response was seen in either strain after administration of PA alone (data not shown). Various attempts to replenish the nasal regions of $W / W^{v}$ mice with cultured MCs were unsuccessful; therefore, data involving reconstituted $W / W^{\nu}$ mice are not available. Collectively, these data indicate that $c 48 / 80$ mediates T-dependent immune responses after nasal administration, and, as in the footpad model, MCs are required for the induction of humoral immune responses. Therefore, regardless of the route, MC

Figure 3 Adjuvant effect of nasally instilled c48/80 is mediated through activation of MCs in the nasal passages. (a) Cross-section through a mouse snout showing both NALTs $(N)$ and palate $(P)$. L, lumen of the nasal cavity. The boxed inset on left is magnified on the right. MCs are stained purple with toluidine blue; representative examples are marked with arrows. Scale bars, $500 \mu \mathrm{M}$ (left) and $50 \mu \mathrm{M}$ (right). (b) Quantification of MC numbers in the palates of mice nasally treated with saline or $32 \mu \mathrm{g} c 48 / 80$ plus PA for 1 h. ${ }^{*} P<0.05$ compared with saline. Data are representative of two independent experiments. (c) Localization of DCs in the T-cell zones of the NALT after nasal instillation of c48/80 plus PA or of PA alone. Sections were stained with anti-CD11c as a marker for DCs (green) and anti-CD90.2 as a marker for T cells (red). Regions of colocalization appear as yellow.

Scale bars, $50 \mu \mathrm{M}$. (d) GMT of serum IgG in WT and $W / W^{v}$ mice after immunization with $c 48 / 80$ and PA. Times on the $x$ axis are those of serum collection after immunization. ${ }^{*} P=0.03$, WT compared with $W / W^{v}$. All error bars, mean + s.d.

activators act as powerful adjuvants capable of initiating specific immune response to pathogen products.

\section{$c 48 / 80$ is a potent mucosal adjuvant}

To further characterize the adjuvant properties of MC activators, we compared the efficacy of various doses of c48/80 to cholera toxin, currently considered the gold standard mucosal adjuvant ${ }^{18,23}$. Mice were challenged nasally with PA alone or with increasing concentrations of c48/80 on days 0,7 and 14, and then bled on day 21 or 22 unless otherwise indicated. All doses of c48/80 tested resulted in higher titers of antigen-specific IgG as compared to PA alone (Fig. 4a). Additionally, antibody titers from mice immunized with the doses of $c 48 / 80$ from $125 \mu \mathrm{g}$ to $8 \mu \mathrm{g}$ were comparable to or greater than titers from mice immunized with cholera toxin. No significant difference in isotype titers was observed between $c 48 / 80$ and cholera toxin treatments (data not shown). Heightened PA-specific serum IgG titers were also observed with catestatin (Supplementary Fig. 3 online). Notably, increased PA-specific serum IgG titers in mice nasally immunized with c48/80 plus PA were maintained for at least 6 months (Fig. $\mathbf{4 b}$ ), and these responses were dependent on neither antigen (including diphtheria toxin or FimH antigen from uropathogenic Escherichia coli) nor mouse strain (Supplementary Fig. 4a,b online).

As previously noted, mucosal immunization can induce the production of secretory IgA at mucosal sites. Therefore we investigated whether $c 48 / 80$ had this effect. Mucosal secretions from sites proximal to the site of vaccine administration (saliva), as well as more distal sites (vaginal washings and fecal extracts) were collected from immunized mice on day 21 and assayed. In all mucosal sites tested, mice treated
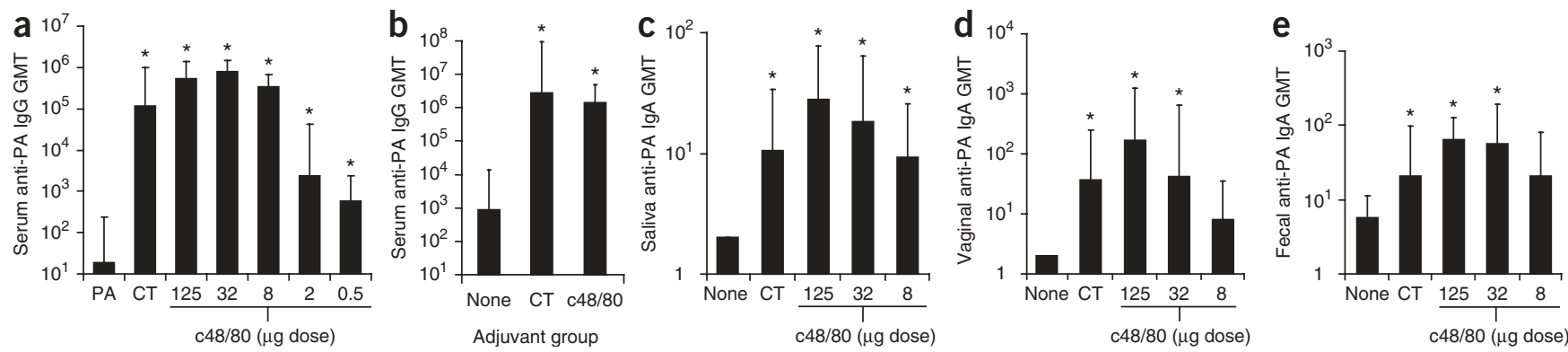

Figure $4 c 48 / 80$ induces a potent and long-lived IgG and IgA response. (a) GMT of PA-specific serum IgG 3 weeks after the first nasal immunization with $\mathrm{PA}$, alone or in combination with cholera toxin (CT) $(1 \mu \mathrm{g})$ or decreasing doses of $c 48 / 80 . n=5$ mice per group; ${ }^{*} P<0.01$ compared with antigen alone. (b) GMT of PA-specific serum IgG on day 175 after immunization with PA alone or in combination with CT ( $1 \mu \mathrm{g})$ or c $48 / 80(8 \mu \mathrm{g})$. ${ }^{*} P<0.02 \mathrm{compared}$ with antigen alone; $n=5$ mice per group. (c-e) GMT of salivary (c), vaginal (d) and fecal (e) PA-specific lgA 3 weeks after the first immunization with $\mathrm{PA}$, alone or in combination with CT $(1 \mu \mathrm{g})$ or decreasing doses of $\mathrm{c} 48 / 80$. ${ }^{*} P<0.05$ compared with antigen alone. All error bars, mean $+\mathrm{s} . \mathrm{d}$.; $n=5$ mice per group. 
Figure 5 c48/80-induced humoral responses confer protection against lethal challenge. (a) Serum from mice immunized with PA or c48/80 was diluted 1:64 and incubated with PA in vitro. This was then combined with anthrax lethal factor to form LeTx, the effective toxin of anthrax, and incubated with macrophages. LeTx killing was assessed as percentage viability compared to cells without LeTx. ${ }^{*} P<0.01$ compared with antigen alone. Error bars, mean + s.d.; serum from $n=5$ mice per group. (b,c) At day 98 , ten mice immunized with either B5R or $B 5 R$ plus $c 48 / 80$ were challenged nasally with

$1 \times 10^{5}$ PFU of vaccinia virus and monitored daily for weight loss (b). Mice were put to death when they had lost $15 \%$ of their original body weight. This data was plotted as percentage survival (c). ${ }^{*} P=0.0325$ using Fisher's exact test to compare the survival of mice immunized with B5R against mice immunized with B5R plus c48/80.

with $c 48 / 80$ or with cholera toxin showed comparable PA-specific IgA levels that were significantly higher than those in mice vaccinated without adjuvant (Fig. 4c-e). The relatively low IgA antibody titers seen in mucosal secretions compared to IgG serum titers are typical and attributable to the dilution effect of mucosal fluids ${ }^{24}$. Thus, after nasal administration, c48/80 was an effective inducer of IgA responses at various mucosal sites. Collectively, these data suggest that administration of a MC activator along with peptide or subunit antigens can induce humoral responses that are at least as vigorous and long-lasting as those induced by cholera toxin, the most effective mucosal adjuvant described to date. It should be noted that these responses were not due to the effect of contaminating Toll-like receptor (TLR) ligands, as preparations of MC-activating compounds did not activate reporter cell lines overexpressing specific TLRs (Supplementary Fig. 5a online). Furthermore, MC activators were able to elicit humoral responses in TLR4 mutant $\mathrm{C} 3 \mathrm{H} / \mathrm{HeJ}$ mice that were comparable to those in WT $\mathrm{C} 3 \mathrm{H} / \mathrm{HeN}$ mice (Supplementary Fig. 5 b). We also found that $c 48 / 80$ elicited similar responses in $M y d 88^{-1-}$ mice and WT C57BL/6 controls (Supplementary Fig. 5c). Thus, MC activators can act as potent adjuvants for the induction of both systemic and mucosal immunity by mechanisms independent of TLRs.

\section{c48/80 induces protective humoral immunity}

We also assayed the antigen-specific antibody titers observed after immunization with MC activators for their protective function using an in vitro neutralizing assay featuring the lethal effects of anthrax lethal toxin (LeTx), of which PA is a subunit ${ }^{25,26}$. We found that adding to LeTx the serum of mice immunized with c48/80 plus PA was highly effective in protecting a macrophage cell line from LeTxinduced killing in vitro (Fig. 5a). Furthermore, we used a vaccinia virus challenge model to demonstrate effective in vivo protection after immunization with $c 48 / 80$ plus the $\mathrm{B} 5 \mathrm{R}$ poxvirus protein ${ }^{27}$. Mice immunized with c48/80 plus B5R had an elevated B5R-specific IgG antibody response $(1: 1,589,344$ geometric mean titer (GMT)), whereas B5R alone immunized mice elicited no response. On day 98, all mice were challenged nasally with $1 \times 10^{5}$ plaque-forming units $(\mathrm{PFU})$ of vaccinia virus WR. After 6 d, 100\% of mice immunized with B5R alone showed endpoint $15 \%$ body weight loss and were put to death (Fig. 5b). However, 50\% of mice nasally immunized with B5R plus c48/80 were protected against infection with vaccinia virus (Fig. 5c). Therefore, MC activation by means of c $48 / 80$ confers high serum titers of functionally protective antigen-specific IgG antibody.

\section{c48/80 is a nontoxic nasal adjuvant}

Although the preceding results indicated that antigen delivery in the context of MC activation could protect mice against lethal pathogen challenge, it raised the specter of toxicity, either directly or through the binding of IgE antigen to FceRI receptors on the surface of MCs, leading to anaphylaxis. Although c48/80 induced mild transient nasal irritation at the highest dose tested, mice did not seem to suffer any other local or systemic side effects characterized by sustained inflammation of the nasal tissue or distal tissues, nor did the mice show any irregular respiration or changes in heart rates at the doses used in this study (data not shown). Furthermore, we did not observe any staining in the upper airways or in the lung after applying c48/80 with a tracer dye into the nasal passages, indicating that $c 48 / 80$ did not enter the upper airways or the lung (data not shown). We also found that c48/80 did not affect either total or antigen-specific IgE levels, even at the highest dose (Supplementary Fig. 6 online). Finally, we attempted to trigger anaphylaxis as previously described ${ }^{28}$ by instilling $50 \mu \mathrm{g}$ of PA into the peritoneal cavities of mice nasally immunized with c48/80 and PA and found no anaphylaxis nor any signs of distress (data not shown). These data are consistent with human studies that observed that cutaneous application of c48/80 did not induce any serious longterm side effects ${ }^{29,30}$ and demonstrate that MC activation in our studies as well seemed to have no notable adverse effects.

\section{DISCUSSION}

The present study demonstrated a heretofore unknown role of the MC in inducing a protective, antigen-specific humoral immune response. Notably, we found that the injection of small-molecule MC activators was sufficient to induce potent and long-lasting humoral responses against protein subunit antigens. It is noteworthy that all MC activating compounds that we examined could induce strong humoral responses when applied nasally (data not shown). MC activators were not able to elicit optimal antibody responses to antigen in $W / W^{v}$ mice, compared to those observed in WT controls. Optimal antibody responses could be restored in these mice by reconstituting the injection site with WT MCs but not $\operatorname{Tnf}^{-1-} \mathrm{MCs}$, indicating the importance of MCs and of MC-derived TNF in mediating the adjuvant activity of MC-activating compounds.

MCs seem to mediate the adjuvant activity of MC activators by inducing the enhanced mobilization of DCs to DLNs, in part by inducing the expression of the lymph node chemokine homing receptor CCR7 on tissue resident DCs. The expression of this molecule on DCs is absolutely required for their mobilization from the peripheral tissues to the $\mathrm{DLN}^{5,31}$. It is likely that MCs accomplish this through the release of proinflammatory mediators, such as TNF, CD40 ligand, or prostaglandin E-2, all known to be produced by MCs and also shown to induce or enhance the expression of CCR7 on cultured $\mathrm{DCs}^{32,33}$. The importance of MCs in the adjuvant activity of MC activators is also underscored by the observation that none of the 
adjuvant activity of MC activators was due to contaminating TLR ligands. For instance, we found that $c 48 / 80$ induced comparable humoral responses in $M y d 88^{-1-}$ and WT controls, and MC-activating compounds did not activate TLRs in vitro. Notably, these data also suggest that MC-dependent adjuvant activity operates independently of conventional signaling pathways such as those mediated through TLRs.

In light of the fact that MCs reside at nearly all host-environment interfaces ${ }^{34}$, our findings demonstrate that MCs function as an integral, sensory arm of the immune system, capable of activating both the innate and adaptive immune systems to clear active infection and to provide lasting protection against subsequent challenges. These results are important at a time when bioterrorism and emerging infectious agents have sparked a renewed interest in the identification of appropriate vaccine candidates that can provide effective humoral protection, especially along mucosal barriers likely to be the entry point for many pathogens ${ }^{15}$. A chief impediment to these efforts is the lack of effective adjuvants; those in existence have limited effectiveness and are known to have long-term associated toxic effects ${ }^{35}$. Although most adjuvants have been developed on a random basis with little information on their mechanisms of action, our findings illuminate the MC as a possible target for vaccine adjuvants, including, but not limited to, the small-molecule MC secretagogues used here. It is noteworthy that because of the abundance of MCs within nasal mucosal surfaces, MC activators can be used through nasal instillation, providing a suitable needle-free alternative for future vaccine development.

\section{METHODS}

Mice. Female CB6F1/Cr, BALB/c, C57BL/6, C3H/HeN, C3H/HeJ and SwissWebster mice were purchased from the US National Cancer Institute-Frederick Animal Production Area. Male MC-deficient (WBB6F1 $W / W^{\nu}$ ) and congenic littermate control mice (WBB6F1 WT) were purchased from Jackson Laboratories. MC-deficient mice were reconstituted with MCs as previously described ${ }^{3}$ (Supplementary Methods online). TNF-deficient mice on a C57BL/6 background were purchased from B\&K Universal. Mice were housed in the Duke University vivarium and provided food and water ad libitum. All experiments were performed according to protocols approved by the Duke Division of Laboratory Animal Resources and the Duke University institutional animal care and use committee.

Immunizations. Mice were nasally immunized according to previously established protocols with slight modifications ${ }^{36}$. Briefly, mice were anesthetized with isoflurane. Antigen or antigen plus adjuvant was administered in a total volume of $15 \mu \mathrm{l}$ of ultrapure $0.9 \%$ saline at $7.5 \mu \mathrm{l}$ per nostril. PA, diphtheria toxin and cholera toxin were obtained from List Biological Labs. c48/80 was prepared as a stock solution in saline. All final dilutions were made in saline. For footpad immunizations, $0.5 \mu \mathrm{g}$ recombinant PA, with or without $32 \mu \mathrm{g}$ $c 48 / 80$ in saline, was injected in the footpad. For DC-recruitment and DLN-hypertrophy studies, footpads were injected with $32 \mu \mathrm{g} c 48 / 80,1 \mu \mathrm{M}$ catestatin or $10 \mu \mathrm{M} \mathrm{MCD}$ in a $20 \mu \mathrm{l}$ volume.

Confocal microscopy. Tissue section preparation is described in Supplementary Methods. Confocal images of tissue sections were obtained with a Nikon Confocal Laser Scanning Instrument containing an argon 488-nm laser, a He-Ne 543-nm laser and a He-NE 633-nm laser. Images were obtained using a Plan Fluor $\times 20$ oil objective and EZ-C1 Nikon software with a channel-series approach to prevent spectral overlap between fluorescent signals. Threshold values for background fluorescence were determined using appropriate isotypematched control-stained sections.

Flow cytometry. DLN, NALT and footpad single-cell preparations were prepared as described in Supplementary Methods. To detect CCR7, samples were initially labeled with anti-CCR7 (Clone EBI-1)(eBioSciences), at $37^{\circ} \mathrm{C}$ for $30 \mathrm{~min}$. Samples were then cooled to $4{ }^{\circ} \mathrm{C}$ and labeled with a polyclonal, phycoerythrin-labeled $\mathrm{Fab}_{2}$ antibody to rat IgG (anti-rat IgG; eBioSciences). To detect DCs, samples lastly were labeled with phycoerythrin-Cy5-labeled
anti-MHC class II (clone M5/114.15.2, eBioSciences) and with allophycocyanin- or FITC-labeled anti-CD11c (clone HL3, BD BioSciences).

Sectioning and staining of mouse snouts. Mouse snouts were prepared as described in Supplementary Methods. We cut $10-\mu \mathrm{m}$ frozen cross sections of the snout on a Leica CM1850 cryotome, air-dried them on slides, and stained them with either $0.5 \%$ toluidine blue in $0.5 \mathrm{M} \mathrm{HCl}$ in PBS or with $0.5 \%$ alcian blue in $0.3 \%$ acetic acid. Alcian blue-stained slides were rinsed with tap water and stained with $0.1 \%$ saffranin $\mathrm{O}$ in $0.1 \%$ acetic acid. All stains were purchased from Sigma. Slides were then air-dried and cover-slipped with Permount (Fisher Scientific) for microscopic examination. Serial sections were made for each treatment group and MCs were enumerated in 8-12 randomly selected sections.

IgG ELISA. We collected samples as described in the Supplementary Methods. Antigen with which mice had been immunized was coated onto 384-well plates at $1-2 \mu \mathrm{g} / \mathrm{ml}$. After blocking, plates were washed and samples were then diluted two-fold and added to the plates to be incubated overnight at $4{ }^{\circ} \mathrm{C}$. Plates were washed and a secondary antibody (specific for total IgG, IgA, or IgG isotypes) coupled to alkaline phosphatase was added to each well and the plate was incubated for $2 \mathrm{~h}$ at $25^{\circ} \mathrm{C}$. Attophos substrate (Promega) was added to each well and incubated for $30 \mathrm{~min}$ at room temperature; the plate was then read on a fluorescence microplate reader at $440 \mathrm{~nm}$ excitation and $570 \mathrm{~nm}$ emission. The end-point titer presented in the results is representative of the reciprocal $\log _{2}$ dilution of the last sample for which the optical density was at least three-fold higher than that of the naive sample. The geometric mean titer and s.d. were calculated using the $\log _{2}$ end-point titers that are then presented as the antilog in figures and tables. Statistics were performed on the $\log _{2}$ titers before conversion. Assessment of IgE titers is described in the Supplementary Methods.

Protection assays. The macrophage protection assay is described in the Supplementary Methods. For the vaccinia challenge, isoflurane-anesthetized mice were challenged by the nasal route with $1 \times 10^{5} \mathrm{PFU}$ vaccinia virus WR (Advanced Biotechnologies) in $30 \mu \mathrm{l}$ sterile PBS (15 $\mu \mathrm{l}$ per nostril). We monitored mice daily and recorded body weights. The end point for infection was a loss of $15 \%$ of the original body weight on the day of infection. Mice were put to death humanely once they reached this end point. Mice that did not lose $15 \%$ of body weight survived the vaccinia challenge. Mice were monitored daily for $14 \mathrm{~d}$.

Statistical analysis. Results were analyzed for statistical significance using the unpaired Student's $t$-test in Microsoft Excel. Differences between groups were considered significant if $P \leq 0.05$. A Fisher's exact test was used to determine whether immunization with B5R plus $c 48 / 80$ provided significantly greater protection against death than immunization with B5R alone.

Note: Supplementary information is available on the Nature Medicine website.

\section{ACKNOWLEDGMENTS}

The authors would like to acknowledge S. Kirwan for technical assistance, K. Owzar for assistance with the statistical evaluation of the immunization data for the vaccinia infection study (that is, protection against weight loss), H.-X. Liao (Laboratory of Protein Expression, Duke Human Vaccine Institute) for the recombinant B5R and M. Jenkins (University of Minnesota) for the CD11c-DTR mice used in this study. The following reagents were obtained through the BioDefense and Emerging Infections Research Repository of the National Institute of Allergy and Infectious Diseases, US National Institutes of Health: anthrax protective antigen (PA), recombinant from B. anthracis, NR-140, and anthrax lethal factor recombinant from B. anthracis, NR-570. This work was supported by US National Institutes of Health grants R37-DK50814, RO1DK077159 and RO1-AI50021.

\section{AUTHOR CONTRIBUTIONS}

J.B.M. and C.P.S. conducted the majority of the experiments; J.P.H. provided discussions and final editing of the manuscript; S.V.P. provided discussion; R.G. assisted with the IgE titers; R.B.-D. provided the NALT micrograph; H.F.S. assisted with the IgG titers and the protection studies and in the design of the study; J.B.M., C.P.S. and S.N.A. designed the study and wrote the manuscript; and the other authors read the paper and commented on the manuscript. 
Published online at http://www.nature.com/naturemedicine

Reprints and permissions information is available online at http://npg.nature.com/ reprintsandpermissions

1. Echtenacher, B., Mannel, D.N. \& Hultner, L. Critical protective role of mast cells in a model of acute septic peritonitis. Nature 381, 75-77 (1996)

2. Malaviya, R., Ikeda, T., Ross, E. \& Abraham, S.N. Mast cell modulation of neutrophil influx and bacterial clearance at sites of infection through TNF- $\alpha$. Nature 381, 77-80 (1996).

3. McLachlan, J.B. et al. Mast cell-derived tumor necrosis factor induces hypertrophy of draining lymph nodes during infection. Nat. Immunol. 4, 1199-1205 (2003).

4. Jawdat, D.M., Albert, E.J., Rowden, G., Haidl, I.D. \& Marshall, J.S. IgE-mediated mast cell activation induces Langerhans cell migration in vivo. J. Immunol. 173, 5275-5282 (2004).

5. Martín-Fontecha, A. et al. Regulation of dendritic cell migration to the draining lymph node: impact on T lymphocyte traffic and priming. J. Exp. Med. 198, 615-621 (2003).

6. Banchereau, J. \& Steinman, R.M. Dendritic cells and the control of immunity. Nature 392, 245-252 (1998).

7. McHeyzer-Williams, L.J. \& McHeyzer-Williams, M.G. Antigen-specific memory B cell development. Annu. Rev. Immunol. 23, 487-513 (2005).

8. Gelfand, E.W. Inflammatory mediators in allergic rhinitis. J. Allergy Clin. Immunol. 114, S135-S138 (2004).

9. Ferry, X., Brehin, S., Kamel, R. \& Landry, Y. G protein-dependent activation of mast cell by peptides and basic secretagogues. Peptides 23, 1507-1515 (2002).

10. Aridor, M., Rajmilevich, G., Beaven, M.A. \& Sagi-Eisenberg, R. Activation of exocytosis by the heterotrimeric G protein Gi3. Science 262, 1569-1572 (1993).

11. Paton, W.D. Compound 48/80: a potent histamine liberator. Br. J. Pharmacol. 6, 499-508 (1951).

12. Krüger, P.-G., Mahata, S.K. \& Helle, K.B. Catestatin (chromogranin A344-358) stimulates release of histamine from rat pleural and peritoneal mast cells. Ann. NY Acad. Sci. 971, 349-351 (2002).

13. Jung, S. et al. In vivo depletion of $\mathrm{CD} 11 \mathrm{c}^{+}$dendritic cells abrogates priming of $\mathrm{CD}^{+}$ T cells by exogenous cell-associated antigens. Immunity 17, 211-220 (2002).

14. Suto, H. et al. Mast cell-associated TNF promotes dendritic cell migration. J. Immunol. 176, 4102-4112 (2006).

15. Pashine, A., Valiante, N.M. \& UImer, J.B. Targeting the innate immune response with improved vaccine adjuvants. Nat. Med. 11, S63-S68 (2005)

16. Boyaka, P.N. et al. Effective mucosal immunity to anthrax: neutralizing antibodies and Th cell responses following nasal immunization with protective antigen. J. Immunol. 170, 5636-5643 (2003).

17. Staats, H.F. \& Ennis, F.A. Jr. IL-1 is an effective adjuvant for mucosal and systemic immune responses when coadministered with protein immunogens. J. Immunol. 162, 6141-6147 (1999).

18. Yamamoto, S. et al. A nontoxic mutant of cholera toxin elicits Th2-type responses for enhanced mucosal immunity. Proc. Natl. Acad. Sci. USA 94, 5267-5272 (1997).
19. Zuercher, A.W., Coffin, S.E., Thurnheer, M.C., Fundova, P. \& Cebra, J.J. Nasalassociated lymphoid tissue is a mucosal inductive site for virus-specific humoral and cellular immune responses. J. Immunol. 168, 1796-1803 (2002).

20. Welle, M. Development, significance, and heterogeneity of mast cells with particular regard to the mast cell-specific proteases chymase and tryptase. J. Leukoc. Biol. 61, 233-245 (1997).

21. Befus, A.D., Pearce, F.L., Gauldie, J., Horsewood, P. \& Bienenstock, J. Mucosal mast cells. I. Isolation and functional characteristics of rat intestinal mast cells. J. Immunol. 128, 2475-2480 (1982).

22. Pearce, F.L. On the heterogeneity of mast cells. Pharmacology 32, 61-71 (1986).

23. Staats, H.F., Nichols, W.G. \& Palker, T.J. Mucosal immunity to HIV-1: systemic and vaginal antibody responses after intranasal immunization with the HIV-1 C4/V3 peptide T1SP10 MN(A). J. Immunol. 157, 462-472 (1996).

24. Palker, T.J. et al. The V3 domain of SIVmac251 gp120 contains a linear neutralizing epitope. Virology 224, 415-426 (1996).

25. Gaur, R., Gupta, P.K., Banerjea, A.C. \& Singh, Y. Effect of nasal immunization with protective antigen of Bacillus anthracis on protective immune response against anthrax toxin. Vaccine 20, 2836-2839 (2002).

26. Hanna, P. Lethal toxin actions and their consequences. J. Appl. Microbiol. 87, 285-287 (1999).

27. Fogg, C. et al. Protective immunity to vaccinia virus induced by vaccination with multiple recombinant outer membrane proteins of intracellular and extracellular virions. J. Virol. 78, 10230-10237 (2004).

28. Snider, D.P., Marshall, J.S., Perdue, M.H. \& Liang, H. Production of IgE antibody and allergic sensitization of intestinal and peripheral tissues after oral immunization with protein Ag and cholera toxin. J. Immunol. 153, 647-657 (1994).

29. Brunet, C., Bedard, P.M. \& Hebert, J. Analysis of compound $48 / 80$-induced skin histamine release and leukotriene production in chronic urticaria. J. Allergy Clin. Immunol. 82, 398-402 (1988).

30. Dor, P.J. et al. Induction of late cutaneous reaction by kallikrein injection: comparison with allergic-like late response to compound 48/80. J. Allergy Clin. Immunol. 71, 363-370 (1983).

31. Forster, R. et al. CCR7 coordinates the primary immune response by establishing functional microenvironments in secondary lymphoid organs. Cell 99, 23-33 (1999).

32. Dieu, M.C. et al. Selective recruitment of immature and mature dendritic cells by distinct chemokines expressed in different anatomic sites. J. Exp. Med. 188, 373-386 (1998)

33. Scandella, E., Men, Y., Gillessen, S., Forster, R. \& Groettrup, M. Prostaglandin E2 is a key factor for CCR7 surface expression and migration of monocyte-derived dendritic cells. Blood 100, 1354-1361 (2002)

34. Abraham, S.N. \& Arock, M. Mast cells and basophils in innate immunity. Semin Immunol. 10, 373-381 (1998).

35. Lindblad, E.B. Aluminium compounds for use in vaccines. Immunol. Cell Biol. 82, 497-505 (2004).

36. Staats, H.F. et al. Cytokine requirements for induction of systemic and mucosal CTL after nasal immunization. J. Immunol. 167, 5386-5394 (2001). 